\title{
STRUCTURE OF A CLASS OF REGULAR SEMIGROUPS AND RINGS
}

\author{
BY GÉRARD LALLEMENT AND MARIO PETRICH
}

Communicated by E. Hewitt, December 14, 1966

One of the most natural approaches to the study of regular semigroups is to impose restrictions on the partial ordering of their idempotents $(e \leqq f \Leftrightarrow e f=f e=e)$. The principal object of this note is to describe the structure of the classes of regular semigroups whose idempotents form a tree or a unitary tree, respectively (see Definition 1). We determine, among other things, a complete set of invariants, isomorphisms, the group of automorphisms, and congruences of these semigroups. We also consider regular rings whose multiplicative semigroup satisfies conditions $(C)$ or $\left(C_{1}\right)$ and give their structure. The terminology concerning semigroups is that of [2] and concerning p.o. sets of [1]. We consider only semigroups with zero; the statements concerning semigroups without zero can then be easily deduced.

Definition 1. A [unitary] tree $T$ is a p.o. set with a unique minimal element 0 [and a unique maximal element 1 ],$T \neq\{0\}$, satisfying

(i) all elements [different from 1] are of finite height;

(ii) every element different from 0 [and different from 1] covers exactly one element.

Definition 2. A regular semigroup whose p.o. set of idempotents is a tree [unitary tree] is said to be J-regular [ $3_{1}-$ regular].

A $\Im_{1}$-regular semigroup has an identity element. In order to find the structure of such semigroups, we need the following construction. For any semigroup $S$ with zero, we write $S^{*}=S \backslash 0$.

Let $T$ be a tree; to every $\alpha \in T^{*}=T \backslash 0$ associate a semigroup $S_{\alpha}$ with zero $0_{\alpha}$; the semigroups $S_{\alpha}$ are pairwise disjoint. If $\alpha \chi>1$ ( $\alpha \chi$ is the height of $\alpha$ in $T$ ), associate to $\alpha$ a partial homomorphism $\phi_{\alpha}: S_{\alpha}^{*} \rightarrow S_{\alpha}^{*}(\bar{\alpha}$ is the unique element of $T$ covered by $\alpha)$. On the set $V=\left(\cup_{\alpha \in T^{*}} S_{\alpha}^{*}\right) \cup 0$, multiplication is defined by induction on the height of $\alpha \in T$ as follows. Let 0 act as the zero of $V$. If $\alpha \chi=\beta \chi=1$ and $x \in S_{\alpha}^{*}, y \in S_{\beta}^{*}$ (multiplication in $S_{\alpha}$ is denoted by juxtaposition), let

$$
\begin{array}{rlrl}
x \circ y & =x y & & \text { if } \alpha=\beta, x y \neq 0_{\alpha}, \\
& =0 & \text { if } \alpha=\beta, x y=0_{\alpha} \text { or } \alpha \neq \beta .
\end{array}
$$

Supposing that multiplication has been defined for all $u \in S_{\gamma}^{*}, v \in S_{\delta}^{*}$, $\gamma \chi, \delta \chi<n(n>1)$, for $x \in S_{\alpha}^{*}, y \in S_{\beta}^{*}$ with $\alpha \chi, \beta \chi \leqq n$, let 


$$
\begin{aligned}
x \circ y & =x y, \quad \text { if } \alpha=\beta, x y \neq 0_{\alpha}, \alpha \chi=n, \\
& =x \phi_{\alpha} \circ y \phi_{\beta}, \quad \text { if } \alpha=\beta, x y=0_{\alpha} \text { or } \alpha \neq \beta, \alpha \chi=\beta \chi=n, \\
& =x \phi_{\alpha} \circ y, \quad \text { if } \alpha \chi=n, \beta \chi<n, \\
& =x \circ y \phi_{\beta}, \quad \text { if } \alpha \chi<n, \beta \chi=n .
\end{aligned}
$$

Under this multiplication, $V$ is a semigroup called a tree of semigroups $S_{\alpha}$ with support $T$; we write $V=\left(T ; S_{\alpha}, \phi_{\alpha}\right)$. Using ideal extensions, a proof by induction on the height of idempotents establishes the following theorem, which is fundamental for our study.

THEOREM 1. A semigroup $V$ is J-regular if and only if $V$ is a tree of completely 0-simple semigroups. If $V=\left(T ; S_{\alpha}, \phi_{\alpha}\right)$, then the set $\left\{T, S_{\alpha}, \phi_{\alpha}\right\}$ constitutes a complete set of invariants of $V$ (i.e., if also $V^{\prime}=\left(T^{\prime} ; S_{\alpha^{\prime}}, \phi_{\alpha^{\prime}}\right)$, then $V \cong V^{\prime}$ if and only if (a) there is an order isomorphism $\xi$ of $T$ onto $T^{\prime}$, (b) for every $\alpha \in T^{*}$, there is an isomorphism $\eta_{\alpha}$ of $S_{\alpha}$ onto $S_{\alpha \xi}^{\prime}$ such that if $\alpha \chi>1$, then for every $x \in S_{\alpha}^{*}, x \phi_{\alpha} \eta_{\bar{\alpha}}=x \eta_{\alpha} \phi_{\alpha \xi}^{\prime}$ ).

Let $S=\left(T_{0} ; S_{\alpha}, \phi_{\alpha}\right)$ be a J-regular semigroup and let $G$ be a group. For each $\alpha \in T_{0}^{*}$, let $\Phi_{\alpha}$ be a homomorphism of $G$ into the group $U_{\alpha}$ of units of the translational hull $\Omega\left(S_{\alpha}\right)$ of $S_{\alpha}$; write $g \Phi_{\alpha}$ $=\left(\lambda_{\alpha}^{o}, \rho_{\alpha}^{o}\right.$ ) (pair of linked left and right translations of $S_{\alpha}$, where $\lambda_{\alpha}^{o}$ and $\rho_{\alpha}^{o}$ are written as operators on the left and right, respectively). Suppose that the diagram

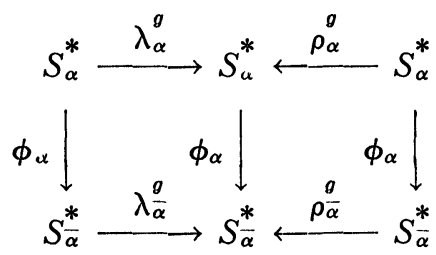

commutes for every $g \in G, \alpha \chi>1$. Denoting multiplication in $S$ and $G$ by juxtaposition, define multiplication on the set $\Sigma=S \cup G$ by

$$
\begin{aligned}
& x \circ y=x y, \quad \text { if } x, y \in S \text { or } x, y \in G, \\
& =x \rho_{\alpha}^{y}, \quad \text { if } x \in S_{\alpha}^{*}, y \in G \text {, } \\
& =\lambda_{\alpha}^{x} y, \quad \text { if } x \in G, y \in S_{\alpha}^{*} \text {, } \\
& =0, \quad \text { if } x=0 \text { or } y=0 \text {. }
\end{aligned}
$$

Write $\Sigma=\left(T ; S_{\alpha}, \phi_{\alpha} ; \Phi_{\alpha}\right)$ where $1 \notin T_{0}, T=T_{0} \cup 1, S_{1}=G$. Letting $1>\alpha$ for all $\alpha \in T_{0}, T$ becomes a unitary tree.

THEOREM 2. Under this multiplication, $\Sigma$ is a $\jmath_{1}$-regular semigroup and the set $\left\{T, S_{\alpha}, \phi_{\alpha}, \Phi_{\alpha}\right\}$ constitutes a complete set of invariants of $\Sigma$. Conversely, every $J_{1}$-regular semigroup can be constructed in this fashion. 
Isomorphisms between two $\Im_{1}$-regular semigroups can be conveniently expressed using matrices. If $\Sigma=\left(T ; S_{\alpha}, \phi_{\alpha} ; \Phi_{\alpha}\right)$ is $J_{1}$-regular, let $M$ be the $T^{*} \times T^{*}$-matrix, $M=\left(m_{\alpha \beta}\right)$ where

$$
\begin{aligned}
m_{\alpha \beta} & =\phi_{\alpha}, \quad \text { if } \beta=\bar{\alpha}, \alpha \neq 1, \alpha \chi>1, \\
& =\Phi_{\beta}, \quad \text { if } \alpha=1, \beta \neq 1, \\
& =0, \quad \text { otherwise. }
\end{aligned}
$$

The semigroup $\Sigma$ is completely determined by semigroups $S_{\alpha}$ and the matrix $M$; we write $\Sigma=\left(M ; S_{\alpha}\right)$.

CoRollary. In order that the $J_{1}$-regular semigroups $\Sigma=\left(M ; S_{\alpha}\right)$ and $\Sigma^{\prime}=\left(M^{\prime} ; S_{\alpha^{\prime}}^{\prime}\right)$ be isomorphic it is necessary and sufficient that there exists an invertible matrix $A$ whose nonzero elements are isomorphisms of $\Omega\left(S_{\alpha}\right)$ onto $\Omega\left(S_{\beta^{\prime}}^{\prime}\right)$ and such that $M A=A M^{\prime}(\Omega(S)$ is the translational hull of $S$ ).

This corollary yields also the group of automorphisms of a $\Xi_{1}$-regular semigroup $\Sigma=\left(M ; S_{\alpha}\right)$. In fact, the group of automorphisms of $\Sigma$ is isomorphic to the group of matrices $A$ described in the corollary with $M A=A M$.

J-regular semigroups can also be characterized in terms of ideals and subdirect products as follows.

Theorem 3. A semigroup $S$ is J-regular if and only if $S$ is regular, has a zero, $S \neq(0)$, and satisfies:

(i) every principal two-sided ideal I of $S$ is of finite height (in the p.o. set of two-sided ideals of $S$ ) and is quasicompletely prime (i.e., $x y z \in I$ implies $x y \in I$ or $y z \in I$; see [3]);

(ii) $S$ is a subdirect product of completely 0 -simple semigroups.

A nonzero homomorphic image of a $J_{1}$-regular semigroup is again $J_{1}$-regular. Homomorphisms of a 3 -regular semigroup onto another can be found using homomorphisms of its support (which is a semilattice) and of different $S_{\alpha}$; these homomorphisms must satisfy certain compatibility conditions. Using this and a characterization of congruences $\sigma$ on a $J_{1}$-regular semigroup $S$ such that $S / \sigma$ is a group with zero, congruences on a $J_{1}$-regular $\Sigma=\left(T ; S_{\alpha}, \phi_{\alpha} ; \Phi_{\alpha}\right)$ can be expressed by means of an arbitrary subset $X$ of $T$ containing its minimal element 0 , congruences on $S_{\alpha}$ for $\alpha \neq 0, \alpha \in X$, satisfying some compatibility conditions.

All the statements above have their analogues for 3-regular semigroups.

When considering rings whose multiplicative semigroup is 3 - or $J_{1}$-regular, we obtain the structure of a somewhat larger class of regu- 
lar rings. On a ring $R$ consider the conditions:

(C) for all idempotents $e, f, g \in R, e<f, g<f$ implies $e$ and $g$ are comparable;

$\left(\mathrm{C}_{1}\right) R$ has a unit 1 and for all idempotents $e, f, g \in R, e<f, g<f$ implies $e$ and $g$ are comparable or $f=1$.

Theorem 4. Let $R$ be a regular ring, $R \neq(0)$. Then

(i) $R$ satisfies (C) if and only if $R$ is a division ring;

(ii) $R$ satisfies $\left(\mathrm{C}_{1}\right)$ if and only if $R$ is either a division ring, or a direct sum of two division rings, or the ring of $2 \times 2$ matrices over a division ring.

Examples of the semigroups studied can be easily found among different semigroups of (partial) transformations on a set, semigroups of endomorphisms of a linear manifold, etc. A (very) special case of a 3 -regular semigroup is an orthogonal sum of completely 0 -simple semigroups [3].

\section{REFERENCES}

1. G. Birkhoff, Lattice theory, Amer. Math. Soc. Colloq. Publ., Vol. 25, Amer. Math. Soc., Providence, R. I., 1948.

2. A. H. Clifford and G. B. Preston, The algebraic theory of semigroups, Vol. I, Math. Surveys, No. 7, Amer. Math. Soc., Providence, R. I., 1961.

3. G. Lallement and M. Petrich, Décompositions I-matricielles d'un demi-groupe, J. Math. Pures Appl. 45 (1966), 67-117.

The Pennsylvania State University 